\title{
Harassment Monitoring System Using Android Smartphone
}

\author{
Gagandeep Singh, Gurjit Singh, and Navdeep Singh
}

\begin{abstract}
In this paper we propose a system for monitoring harassment. It is essentially software installed on phone which informs the security (e.g. police) and dear ones (e.g. parents) with location details and seeking for help message. It posts the same details on server to notify public for help.
\end{abstract}

Index Terms - GPS, location detection, 3G, Android.

\section{INTRODUCTION}

Android is an open mobile platform developed by the Open Handset Alliance (OHA) led by Google, Inc. Its platform consists of several layers: the Linux kernel, native libraries, the Dalvik virtual machine (VM), and an application framework. The Linux kernel provides basic operating system services and hardware abstraction for the upper software stacks. Native libraries support the miscellaneous functionalities of web browsing, multimedia data processing, database access, and GPS reception optimized for a resource-limited hardware environment. The register-based Dalvik VM runs Java code with low memory demand. At the top of the layers, Android provides a component-based programming framework so that users can easily build their own applications [1].

An Android application is written with new and reusable application building blocks (example: activity); broadcast intent receiver, service, and content provider. After an application is written, it is deployed as .apk file (Android package file). .apk file contains codes, resources, and a special XML file called the Android Manifest file. This contains basic information about an application such as the package name, component descriptions, and permission declarations. Harassment monitoring system adopts a mobile cell phone network. Based on the experiences and findings of the field experiments, a new monitoring system is proposed here.

The system has the following requirements. Easy to implement and add functions, able to manage many numbers efficiently, Adaptive for mobility of user and Low cost. To satisfy the above requirements, the proposed system adopts $3 \mathrm{G}$ communication function and collects user's information using Global positioning system. In addition cloud technique is adopted for storing and retrieving user's details such as call and user's location. This system consists of telephony manager for identifying the information about the Android mobile terminal which each user holds, and the server which

Manuscript received August 9, 2013; revised December 18, 2013.

The authors are with Department of CSE, Sri Sai Institute of Engineering and Technology, Amritsar (e-mail: er.gagan29@gmail.com). stores user's information. The Collected information in this system contains the position and time information of android mobile terminal. When the user calls the security (e.g. police) or a parent, an immediate alert message will be sent to the police's and parent's phone. With this system it is possible for security and parents to track the location of the user. Android is a software stack for mobile devices with an operating system, middleware and key applications. Its SDK provides tools and APIs necessary to begin developing applications on the Android platform using the Java programming language.

\section{A. Android Architecture}

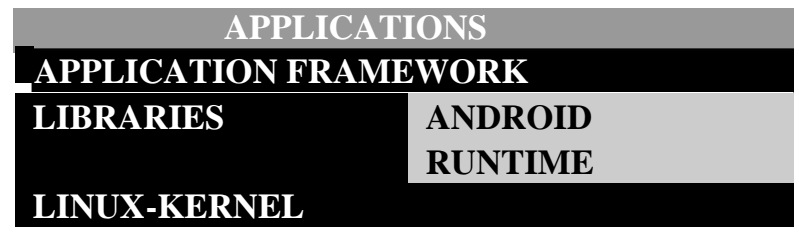

Android relies on Linux version 2.6 for core system services. The kernel also acts as an abstraction layer between the hardware and the rest of the software stack. Every Android application runs in its own process, with its own instance of the Dalvik Virtual machine [2]. The VM is register-based, and runs classes compiled by a Java language complier that have transformed into the dex format by the included "dx" tool. Android uses SQLite which is a powerful relational database engine available to all applications. Rich development environment includes a device emulator, tools for debugging, memory and performance profiling, and a plugin for the Eclipse IDE.

\section{EXISTING SySTEM}

In the existing system the monitoring is done by fixing tags in different location for identifying the exact position. The android terminal is connected to Bluetooth and wireless LAN and it is limited to shorter distance. The proposed system has no boundary limits. The communication link to the management server is managed by wireless LAN which is relatively slow when compared to the $3 \mathrm{G}$ network [3], [4]. The dynamic pairing of mobile terminal is mandatory. The network is more complex and it is not reliable. The message is transferred through wireless LAN and it is not secure.

\section{A. Drawbacks of Existing System}

A person cannot know the user's (victim's) current location.

There is a possibility of data loss during the message 
transfer from one mobile terminal to another mobile Terminal. There is a lag in data transfer due to $2 \mathrm{G}$ network. Public tending to offer help cannot know about victim's location.

\section{PROPOSED SYSTEM}

Different functions have been implemented for the new generation monitoring system such as telephony manager to track outgoing call and sms. Android mobile terminal is connected to high speed $3 \mathrm{G}$ network for effective data transfer. Monitoring can be made at a very high speed without any distortion in the network. This proposed system makes use of the cloud technology to store and retrieve telephony information using SOAP protocol. Global Positioning System, shortly known as GPS System, is the system that enables you to know the location of the victim. It consists of minuscule chip which is attached to the object to be tracked. This chip will give out signals which are tracked by the satellite which sends data to the earth giving the exact location of the user. GPS tracking has come to be accepted on a global scale. Due to the usage of $3 \mathrm{G}$ network the data is retrieved and stored in the server at a very high speed.

\section{A. Features of the Proposed System}

One can easily get to know that the user needs help. It also brings the current location of the user (victim). These alert messages are sent to the security and parent's mobile as a SMS format. The public can also view and offer help with the help of the cloud service in which they can track the position of the victim at any time. It is possible to track the exact position of the victim with the help of Google Maps; with the help of latitude and longitude values it is possible to locate any position (see Fig. 1).

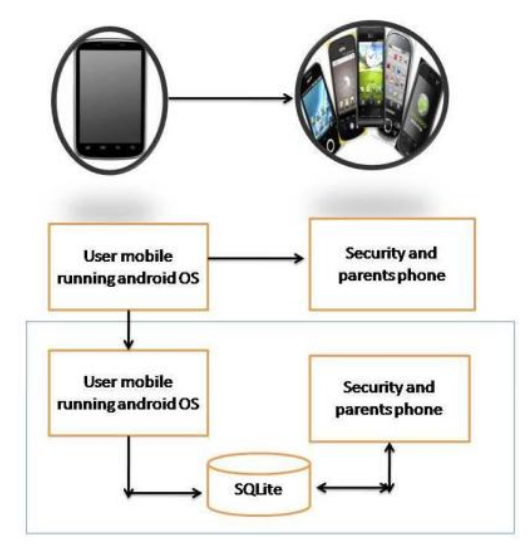

Fig. 1. Architecture of proposed system.

\section{ANALYSIS}

As we are using Android as an illustrative example, we begin by describing several attack vectors possible on that platform. In the course of paper, we demonstrate how the security architecture addresses the security problems. First, let's briefly explain the basic Android security concepts.

\section{A. Android Security}

Android is a Linux platform for mobile phones with a Java middleware on top of the OS. Android applications are usually Java-based, although native code can also be accessed through the Native Development Kit (NDK). Android has two parts of security enforcement. First, applications run as Linux users and thus are separated from each other. A security hole in one application does not affect other applications. However, there is also a concept of inter-process communication (IPC) between different applications, or between the Android components of the applications such as activities and services. The Java-based Android middleware implements a reference monitor to mediate access to application components based upon permission labels defined for the component to be accessed. Any application requires an appropriate permission label before it can access a component. A number of features further refine Android's security model. One example is the concept of shared user IDs, i.e., different applications can share the same user ID if they are signed by the same developer certificate. Another refinement is protected APIs: Several security-critical system resources can be accessed directly rather than using components [5]. WS-Security (Web Services Security, short WSS) is a flexible and feature-rich extension to SOAP to apply security to web services. It is a member of the WS family of web service specifications and was published by OASIS. This protocol specifies how integrity and confidentiality can be enforced on messages and allows the communication of various security token formats, such as SAML, Kerberos, and X.509. Its main focus is the use of XML Signature and XML Encryption to provide end-to-end security.

\section{B. SOAP}

SOAP, originally defined as Simple Object Access Protocol. It is a protocol specification for exchanging structured information in the implementation of Web Services in computer networks. It relies on Extensible Mark up Language (XML) for its message format, and usually relies on other Application Layer protocols, most notably Hypertext Transfer Protocol (HTTP) and Simple Mail Transfer Protocol (SMTP), for message negotiation and transmission. SOAP can form the foundation layer of web services protocol stack, providing a basic messaging framework upon which web services can be built [6].

\section{IMPLEMENTATION}

\section{A. Hardware and Software}

The Android mobile terminal is Google Dev Phone 1 and 2. The operating system for the terminal is Android 2.3(Ginger bread). We develop mobile ad hoc network software using Java programming language and SDK for Android 2.3.

\section{B. Implementation Functions}

So far, we have implemented communication software to construct a 3G network by GPS for the User tracking

System [7] we took care of security in communication between each pair of mobile terminals using WS-Security. When a mobile terminal communicates with another mobile terminal, it is necessary to establish pairing of such two mobile terminals before their communication occurs. When the user mobile terminal calls a particular security (e.g. police) 
phone number, an immediate alert message is sent to the same security's phone and also to parents phone using $3 \mathrm{G}$ network and simultaneously messages are stored in the online server for public's access.

\section{RESULTS}

This new generation harassment monitoring system is software for phone. When the user makes a call to security or parents, the software starts executing loop wise. Once there is an outgoing call, the function "phone State Listener" determines if the call was to the security or parent's phone number. Once satisfied, the loop executes to send the alert message seeking help with location details. Flow chart of the software execution is shown in Fig. 2.
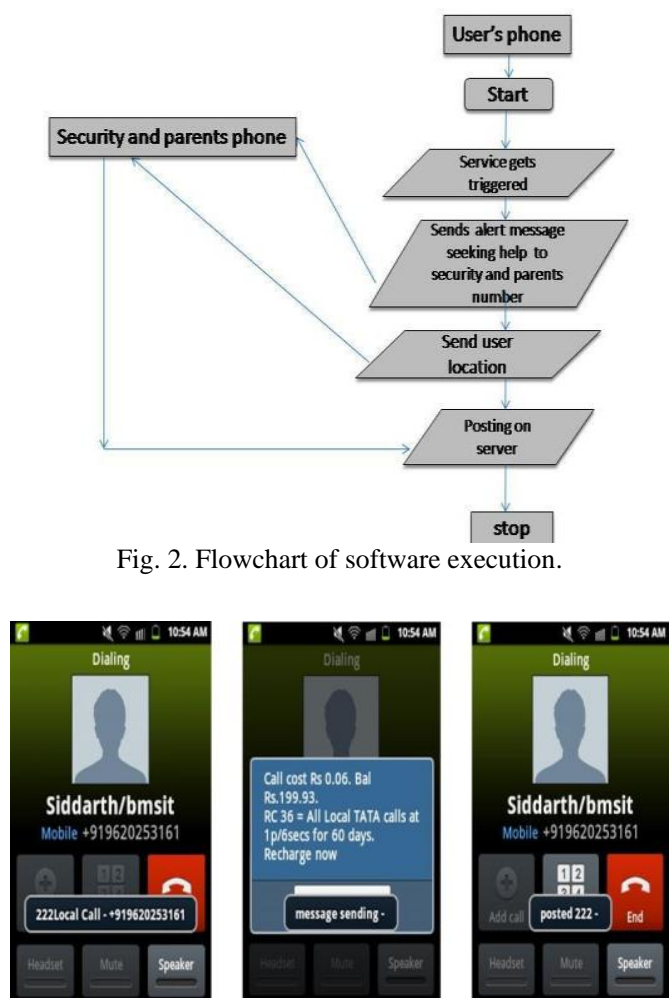

Fig. 3. Snapshots of execution.

The following snapshots in the Fig. 3 show the software execution on an actual android phone with toast messages. Toast message is a notification which ensures the successful execution of respective loops. In the snapshot siddhart/bmsit is the person to whom the user has given call to for help.

1) Toast message "222Local Call-+919620253161" indicates that the function phone State Listener has identified the number for further execution.

2) Toast message "message sending" indicates that the alert message seeking help and his boundary location (latitude and longitude) is sent to Siddhartha/bmsit and also to the security's number registered.

3) Toast message "posted 222-" ensures that the information has been posted on to the web server.

\section{CONCLUSION}

Considering the harassments activities in current world, in this paper, we have implemented the new generation harassment monitoring system and system features to meet the requirements. Using this system it is possible for the user to inform the Security like police and also to parents whenever he/she meets with unexpected circumstances, especially women. More importantly it is not necessary for the security to attend the call, the user just has to press the call button and the software starts its execution. Using telephony manager technique, the proposed new generation harassment monitoring system can adapt to various mobility of user by adjusting network.

\section{REFERENCES:}

[1] I. E. Paul and S. Pusuluri, "Hospital Information Service on Android Mobile Devices," International Journal of Engineering Research and Technology, ISSN: 2278-0181, vol. 1, issue 3, May 2012.

[2] S. B. Komal, R. B. Amit, S. C. Sneha, and B. A. Khivsara, "Android Application using GPS Navigation," Issue of International Journal of Electronics, Communication and Soft Computing Science and Engineering, Berkeley, vol. 5, 2012, pp. 84-89.

[3] A. Kushwaha and V. Kushwaha, "Location Based Services using Android Mobile Opera ting System," International Journal of Advances in Engineering \& Technology, vol. 1, 2011, pp. 14-20.

[4] M. Singhal and A. Shukla, "Implementation of Location Based Services in A ndroid using GPS and Web Services," International Journal of Computer Science Issues, vol. 2, 2012, pp. 237-242.

[5] J. Li, D. Gu, and Y.-H. Luo, "Android Malware Forensics; Reconstruction of Malicious Events," 32 Nd IEEE International Conference on Distributed Computing Systems Workshops, 2012, pp. 552-558.

[6] R. C. Gadri, A. Chavan, R. Sonawane, and S. Kamble, "Land Vehicle Tracking Application on Android Platform," International Journal of Engineering Research and Applications (IJERA), vol. 2, no. 3, pp. 1978-1982, 2012.

[7] A. Doulamis, N. Pelekis, and Y, Theodoridis, "EasyTracker: An Android Application for Capturing Mobility Behaviour," in Proc. 16th Panhellenic Conference on Informatics, 2012, pp. 357-362.

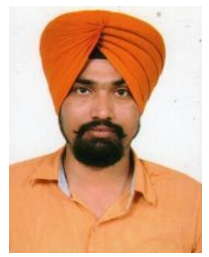

Gagandeep Singh is working as an assist. professor in Department of Computer Science \& Engineering, Sai Institute of Engineering and Technology, Manawala, Amritsar, Punjab, India. He obtained his B.Tech (computer science engineering) from Sri Sai Institute of Engineering \& Technology, Punjab, India, M.Tech (computer science \& engineering from ACET, Manawala, Punjab, India he has 5 years of research and UG \& PG teaching experience. He has 15 research papers to his credit in various international journals and conferences. He is member of International Association of Engineers HONG KONG and International Association of Computer Science \& Information Technology, SINGAPORE. Member of International Journal i-Xplore International Research Journal Consortium (IIRJC). Member of International Reviewer Board of Enhanced Research Publication. Associate Member of UACEE Universal Association of Computer and Electronics Engineers. More than 12 International Papers has been reviewed of different journal. His areas of research and interest include Web Security, Software.

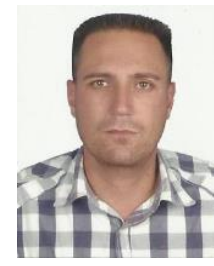

Navdeep Singh is working as an assist. professor in Department of Computer Science \& Engineering, Sai Institute of Engineering and Technology, Manawala, Amritsar, Punjab, India. He obtained his B.Tech (computer science engineering) from Amritsar College of Engineering \& Technology, Punjab, India, M.Tech (computer science \& engineering from ACET, Manawala, Punjab, Presently, India he has 5 years of research and UG \& PG teaching experience. He has 3 research papers to his credit in various international journals and conferences. 